Gut, 1984, 25, 375-380

\title{
Jejunal motility in patients with functional abdominal pain
}

\author{
J G C KINGHAM, R BOWN, R COLSON, AND M L CLARK
}

From the Departments of Gastroenterology, and Medical Electronics, St Bartholomew's Hospital, London, and the Department of Medicine, Frimley Park Hospital, Camberley, Surrey

SUMmaRy A radiotelemetric system using dual pressure pills was used to record the jejunal motility in man. Prolonged recordings were made under relatively physiological conditions. Six patients with functional abdominal pain and six age/sex matched normal subjects were studied. Motility patterns varied markedly both within and between subjects. No differences were detected between the two groups. In particular, the occurrence of characteristic pain in the patients bore no relation to the recorded motor activity. The wide range of jejunal motility patterns in normal subjects needs to be appreciated before conclusions can be drawn about possible abnormalities in gut diseases or disorders.

Despite the development of sophisticated methods for recording electrical and pressure changes within the alimentary tract, our understanding of small bowel motility is limited. The normal pattern in dogs was defined by Szurszewski ${ }^{1}$ and Code $^{2}$ and similar activity has been shown in man. ${ }^{34}$ It seems probable, however, that motor activity is more variable in $\operatorname{man}^{56}$ than dogs and that care should be exercised in the interpretation of supposed abnormalities such as those recently described in a patient with irritable bowel syndrome. ${ }^{7}$

The aims of the present study were twofold. Firstly, we made prolonged recordings of jejunal motor activity in normal subjects under relatively physiological conditions to observe variations within and between subjects during fasting, feeding, and sleeping. Secondly, we made similar recordings in patients suffering from functional abdominal pain in whom a diagnosis of irritable bowel syndrome had been made.

\section{Methods}

SUBJECTS

We studied six patients (three men) with functional abdominal pain whose ages ranged from 19-67 years and six normal subjects who were matched with the patients for age and sex. The protocol for the study

Address for correspondence: Dr J G C Kingham, Dept of Gastroenterology, St Bartholomew's Hospital, London EC1A 7BE.

Received for publication 6 July 1983 was approved by the Ethical Committee of St Bartholomew's Hospital.

Functional abdominal pain was diagnosed on the basis of a typical history and normal findings on physical examination, barium radiology, rectal biopsies, and laboratory tests on faeces and blood. We use the term functional abdominal pain to describe the irritable bowel syndrome in which the dominant symptom was abdominal pain. In addition patients experienced such symptoms as irregular bowel habit, flatulence, sensations of fullness, and incomplete evacuation which confirmed the diagnosis to our satisfaction. The recordings were made using a radiotelemetric system (Rigel Research Ltd, 99 Gander Green Lane, Sutton, Surrey, England). Two pressure sensitive pills (Rigel 7014, length $20 \mathrm{~mm}$, diameter $8 \mathrm{~mm}$ ) were attached $20 \mathrm{~cm}$ apart to a $150 \mathrm{~cm}$ length of radio opaque thread (Fig. 1). The subjects swallowed the pills which were allowed to pass through the stomach into the duodenum. Under radiographic screening the pills were positioned so that the proximal was at the duodenojejunal flexure and the distal $20 \mathrm{~cm}$ further on. The tethering thread was then taped to the subject's cheek, leaving no slack in the stomach, so that the position of the pills remained constant as judged by radiographic screening during the study. An array of radial and coil aerials was wrapped round the patient's abdomen to receive the emitted radio signals. The signals were fed into paired receiving units (Rigel 7040) and the pressures recorded on a dual channel 


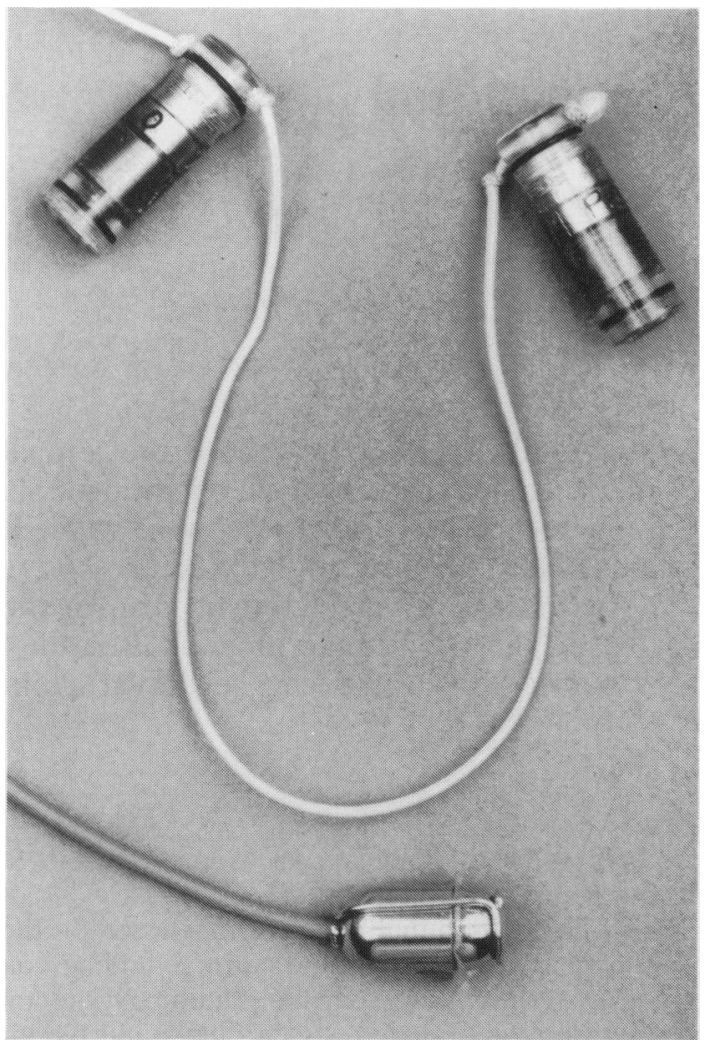

Fig. 1 Two pressurew pills in series. Crosby capsule shown for size comparison.

chart recorder (Servoscribe 460, Smiths Industries).

The study period was 30 hours and started at 9.00 am after an overnight fast. Three standard meals (500 calories, $50 \mathrm{~g}$ carbohydrate, $30 \mathrm{~g}$ protein, $20 \mathrm{~g}$ fat) were eaten six, nine, and 12 hours after the start of the study period (Fig. 2). Nocturnal sleep was variable but most subjects slept from $11.00 \mathrm{pm}$ to
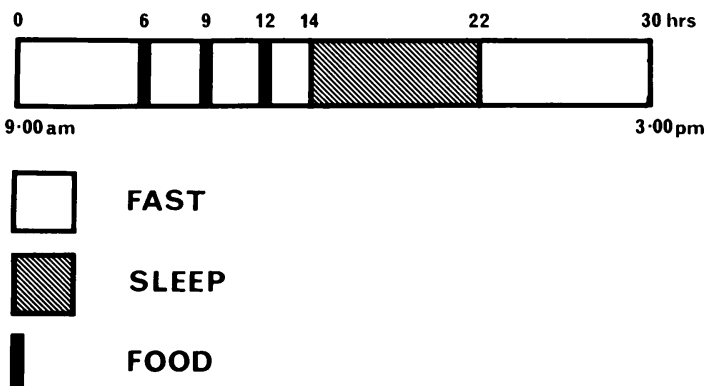

Fig. 2 Sequence of events during 30 hour study period.
$7.00 \mathrm{am}$ the following day. There was then an eight hour fast until the recording was terminated at about $3.00 \mathrm{pm}$ of the second day. The pills were withdrawn by gentle traction on the thread.

The periods of fasting, eating, and sleeping and the occurrence of the patients' characteristic abdominal pain were recorded on the chart. Brief episodes of signal loss occurred during the day in all subjects, but minor repositioning of the aerial array corrected this. Signal loss during sleep was not encountered. Signal loss was recognised as rapid, gross, and irregular deviations from the baseline associated with weak or absent signal strength. Accumulated breaks in recording because of signal loss or visits to the lavatory accounted for less than $5 \%$ of recording time. It is possible, but unlikely, that a brief episode of phase III activity could be obscured by such breaks, though these occurred with equal frequency in patients and controls.

\section{MOTOR ACTIVITY}

A typical section of recording is shown in Figure 3. The lower tracing is the pressure recorded at the proximal site and the upper tracing at the distal site. Two consecutive episodes of regular phase III spike activity $^{2}$ propagated from proximal to distal recording site are shown. A more magnified view of the phase III activity in one channel is shown in Figure 4 . The chart speed was one division $(\mathrm{cm})$ per minute and the calibration one large division (10 small) $=20 \mathrm{~cm}$ of water. The functions of fasting phase III activity measured were: (a) duration at the proximal recording site (min); (b) duration at the distal recording site (min); (c) speed of propagation from proximal to distal site $(\mathrm{cm} / \mathrm{min})$; (d) periodicity - that is, interval between consecutive complexes (min); (e) amplitude = pressure ( $\mathrm{cm}$ of water); (f) frequency (contractions/min).

\section{STATISTICAL ANALYSIS}

The values obtained for the above phase III

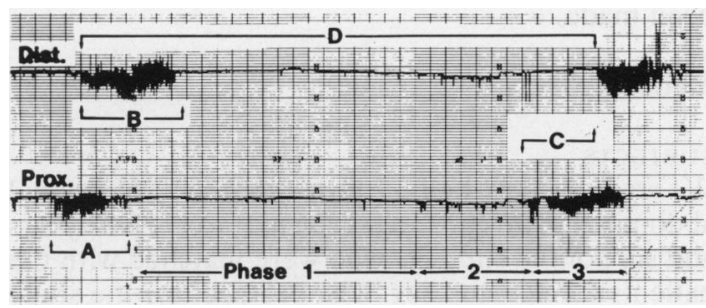

Fig. 3 Typical section of pressure recording showing two propagated episodes of phase III activity. 


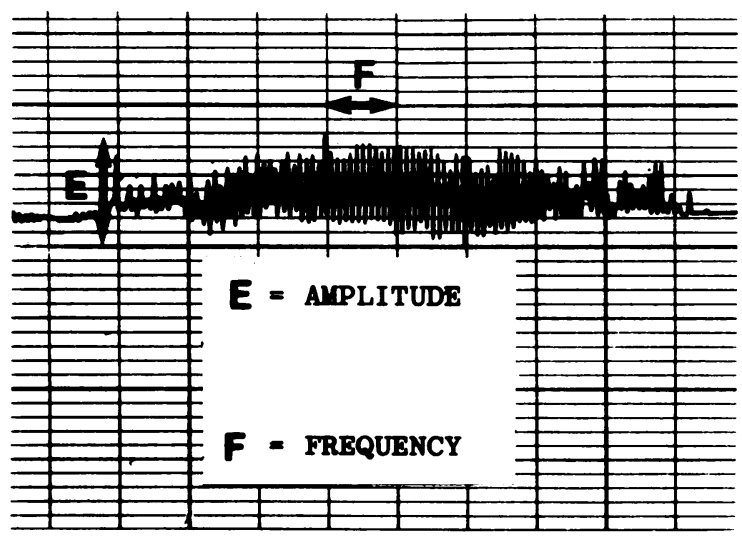

Fig. 4 Magnified view of phase III activity in one channel.

functions were not normally distributed and are expressed as medians with a range. Median values for the six normal control subjects were compared with those of the six functional abdominal pain patients using a Mann-Whitney test.

\section{Results}

There was very marked variability in all phase III functions measured in the normal control subjects with the exception of the contraction frequency which varied little (Table). The variability was both within and between subjects. A similarly wide range of values was observed in functional abdominal pain patients. There were no significant differences between the two groups in respect of any of the phase III functions $(<0 \cdot 2)$. The data were derived from a total of 155 (73 control, 82 functional abdominal pain) episodes of fasting phase III activity. All patients experienced their customary pain at some time during the recording, but there

Table Functions of phase III activity in control subjects and patients with functional abdominal pain (FAP)

\begin{tabular}{|c|c|c|c|c|}
\hline \multirow[b]{2}{*}{ Phase III } & \multicolumn{2}{|l|}{ Control } & \multicolumn{2}{|l|}{$F A P$} \\
\hline & Median & Range & Median & Range \\
\hline Duration prox (min) & 5 & $1-10$ & 5 & $1-9$ \\
\hline Duration dist (min) & 5 & $1-15$ & 5 & $2-12$ \\
\hline Speed propagation & & & & \\
\hline$(\mathrm{cm} / \mathrm{min})$ & 4 & $1-16$ & 5 & $2-11$ \\
\hline Periodicity (min) & 90 & $20-194$ & 82 & $10-152$ \\
\hline Amplitude (cm water) & 17 & $9-80$ & 18 & $8-45$ \\
\hline $\begin{array}{l}\text { Frequency } \\
\text { (contractions/min) }\end{array}$ & 11 & $9-14$ & 11 & $8-13$ \\
\hline
\end{tabular}

was no relationship to phase. III activity. In particular propagated phase III activity was seen to occur while pain was present and its appearance neither abolished nor altered the pain (Fig. 5).

The following observations were made with equal frequency in both controls and patients with functional abdominal pain.

A distal start of phase III activity - that is, recorded at the distal site only - occurred in approximately one in four recorded complexes (Fig. 6). This pattern was haphazard in distribution throughout the 30 hour recording period, but was seen in nine of those studied (four functional abdominal pain patients, five controls).

Failure of propagation of a proximally initiated phase III complex - that is, recorded at proximal site only - occurred in approximately one in 15 recorded complexes (Fig. 7). This too was haphazard throughout the observation period and was seen in seven of the subjects (three functional abdominal pain patients, four controls).

Many (19) of the recorded phase III complexes were bizarre or multiphasic in either or both recording sites. The occurrence of these unusual complexes was inconstant and noted at least once in all subjects (Fig. 8). Retrograde propagation - that is, the complex appearing in the distal before the proximal site - was observed on two occasions, once in a control and once in a patient (Fig. 9).

Food, although always inducing the fed pattern of irregular contractile activity, did not necessarily abolish phase III activity which was sometimes seen superimposed on the fed pattern. This was observed in two controls and one patient (Fig. 10). The interval between onset of eating and the next propagated episode of phase III activity varied markedly within and between individuals but the range was similar in functional abdominal pain patients (median $247 \mathrm{~min}$, range $15-570 \mathrm{~min}$ ) and controls (median $261 \mathrm{~min}$, range $23-710 \mathrm{~min}$ ).

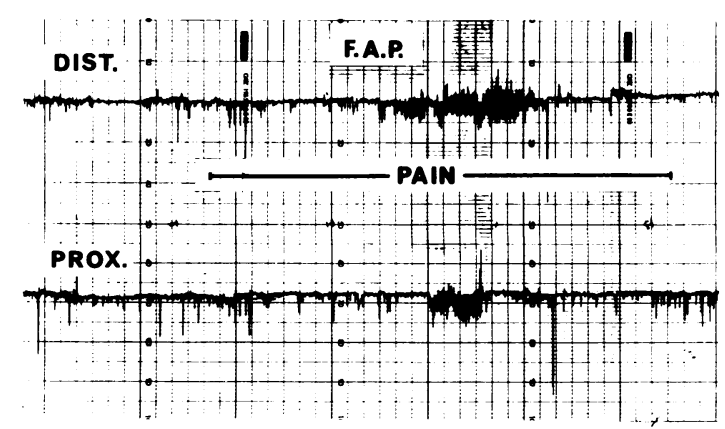

Fig. 5 Phase III activity appearing during pain in patient with functional abdominal pain (FAP). 


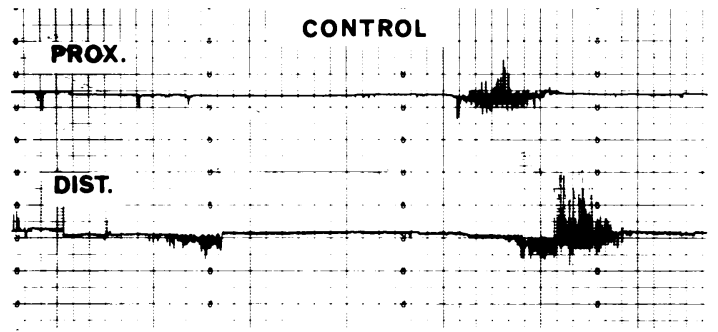

Fig. 6 Distal start of phase III activity in control subject.

None of the above variations from the traditionally accepted 'normal' pattern bore any relation to symptoms in the patients with abdominal pain.

\section{Discussion}

Jejunal motility patterns vary from species to species. Studying fasting dogs Szurszewski, ${ }^{1}$ and Code and Marlett, ${ }^{2}$ showed bursts of regular electrical and concomitant contractile activity which migrated slowly down the small intestine (interdigestive migrating complex or IMC). This regular fasting activity ceased abruptly on feeding to be replaced by frequent bursts of haphazard activity the fed pattern.

Code' $\mathrm{s}^{2}$ classification of fasting motor activity is now generally accepted. The easily recognisable phase of regular spiked activity (phase III) peters out to be replaced by a long period of quiescence (phase I). This quiescence is then interrupted by irregular spiked activity (phase II) which heralds the appearance of the next phase III activity. The conventional view of the interdigestive migrating complex is that it starts in the stomach and migrates down to the terminal ileum at about which time a new interdigestive migrating complex will commence proximally. ${ }^{2}$

Although food interrupts the fasting pattern in dogs this is not the case in rabbits, sheep, or horses. ${ }^{8}$ In rats $^{9}$ food does interrupt the fasting pattern and

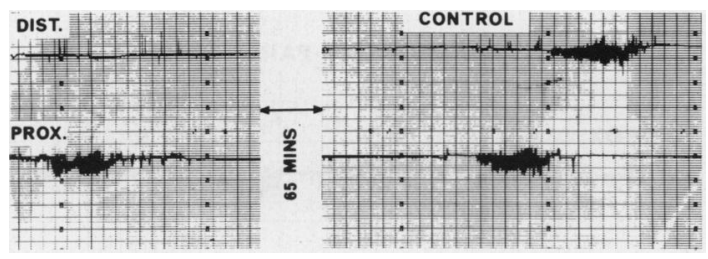

Fig. 7 Failure of propagation of proximally initiated phase III activity in control subject.

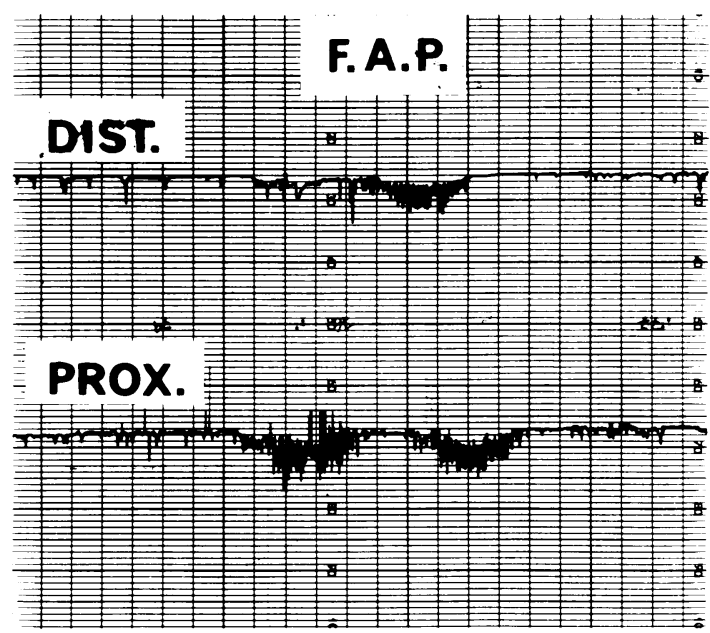

Fig. 8 Multiphasic phase III activity in proximal channel in patient with functional abdominal pain (FAP).

in pigs such interruption is variable depending on the diet. This suggests then that interruption of interdigestive migrating complex by a meal is characteristic of carnivores, but not herbivores, and that omnivores may show either pattern. ${ }^{10}$ In man Vantrappen et $a l,{ }^{4}$ and Thompson et al ${ }^{5}$ have both reported that the fasting activity is interrupted by food. In fact Thompson ${ }^{19}$ has suggested that in man, because of regular feeding, interdigestive migrating complex are probably not present for most of the day, but are mainly a nocturnal phenomenon.

The present study emphasises the wide spectrum of jejunal motility patterns seen in man during prolonged radiotelemetric recordings under relatively physiological conditions. Phase III activity is not only immensely variable in periodicity, duration, and intensity but may start or cease at variable points in the small intestine and is not necessarily abolished by food. Similar variations of phase III patterns in man were recently reported by Kerlin and Phillips though in their studies feeding

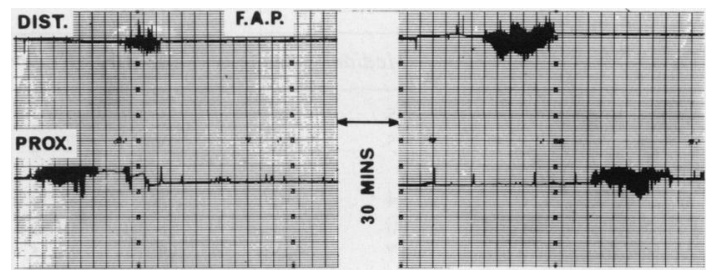

Fig. 9 Anterograde followed by retrograde propagation of phase III activity in patient with functional abdominal pain (FAP). 


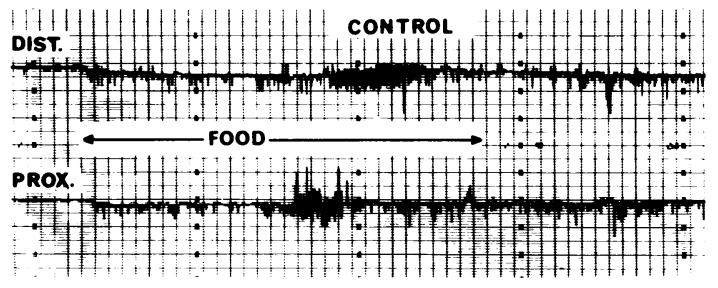

Fig. 10 Phase III activity appearing superimposed on fed pattern in control subject.

always abolished the fasting pattern.

Functional bowel disorders are thought to result from disordered gut motility. Functional abdominal pain may be considered part of the irritable bowel syndrome, though some ${ }^{12}$ feel this latter term should only be applied to symptoms suggesting a colonic disturbance. Many patients, however, with functional abdominal pain do not fulfill all the criteria of classical irritable bowel syndrome. In such patients it is believed that the symptoms may originate from sites proximal to the colon and thus the earlier terms spastic or irritable colon are misleading. In fact evidence incriminating any part of the alimentary tract in causing the symptoms of irritable bowel syndrome has been slow to appear but recently we have reproduced characteristic pain in such patients by balloon distension of the colon ${ }^{13}$ or small intestine. ${ }^{14}$ In some patients distension in either site led to symptoms. There is thus good reason to anticipate disordered function of the small intestine in a proportion of patients with irritable bowel syndrome. Very few studies of small intestinal motility in functional bowel disease have been published.

Horowitz and Farrah ${ }^{15}$ reported irregularities of contractile activity in patients with irritable bowel syndrome over 20 years ago, although they used a freely migrating rather than a tethered pressure sensor. Holdstock et $a l^{16}$ observed bursts of jejunal contractile activity in three patients with unexplained abdominal pain, particularly after meals, but they too used a freely migrating pressure pill.

The use of freely migrating sensors was later abandoned because of inability to record consistent pressure patterns; phase III activity was not detectable, presumably because the sensor was swept along with the complex. Stationary sensors are now generally accepted as standard,${ }^{5}$ though the effect on motility of the tethered sensor itself is unknown.

Thompson $\mathrm{et}_{\mathrm{al}} \mathrm{l}^{7}$ using a tethered pressure sensor recently reported a pattern of jejunal motility in a patient with irritable bowel syndrome which was felt to be abnormal. Fasting motor complexes were less frequent than normal during the day, though normal at night, and pain was associated with irregular activity which ended with the resumption of cyclical fasting activity. Our study, on the other hand, has failed to detect any differences in activity between normal subjects and patients with functional abdominal pain. The difference between our findings and those of Thompson $\mathrm{et} \mathrm{al}^{7}$ is unlikely to be due to technique as very similar systems were used in both studies. We consider the explanation may lie in the very variable nature of the normal pattern of phase III motor activity. If any such abnormality were present in patients with functional abdominal pain it would be undetectable without studying a much larger group of patients for an even longer period. It is possible that these patients may have abnormalities of small bowel motility other than phase III activity, but our technique did not allow accurate quantitation of phase I and II activities.

Our results do not preclude the possibility that patients with another variant of irritable bowel syndrome, predominant diarrhoea for instance, may show recordable motility disturbances.

We conclude that possible abnormalities of jejunal motility in gut disorders should be interpreted cautiously in view of the very wide range found in normal subjects.

\section{References}

1 Szurszewski JH. A migrating electric complex of the canine small intestine. Am J Physiol 1969; 217: 1757-63.

2 Code CF, Marlett JA. The interdigestive myoelectric complex of the stomach and small bowel of dogs. $J$ Physiol 1975; 246: 289-309.

3 Stanciu C, Bennett JR. The general pattern of gastroduodenal motility: 24 hour recordings in normal subjects. Rev Med Chir Soc Med Nat Iasi 1975; 79: 31-6.

4 Vantrappen G, Janssens J, Hellemans J, Ghoos Y. The interdigestive motor complex of normal subjects and patients with bacterial overgrowth of the small intestine. J Clin Invest 1977; 59: 1158-66.

5 Thompson DG, Wingate DL, Archer L, Benson MJ, Green WJ, Hardy RJ. Normal patterns of human upper small bowel motor activity recorded by prolonged radiotelemetry. Gut 1980; 21: 500-6.

6 Kerlin P. Phillips SF. Variability of motility of the ileum and jejunum in healthy humans. Gastroenterology 1982; 82: 694-700.

7 Thompson DG, Laidlow JM, Wingate DL. Abnormal small-bowel motility demonstrated by radiotelemetry in a patient with irritable colon. Lancet 1979; 2: 1321-3. 
8 Grivel ML, Ruckerbusch Y. The propagation of segmental contractions along the small intestine. $J$ Physiol 1972; 227: 611-25.

9 Ruckerbusch Y, Fioramonti J. Electrical spiking activity and propulsion in small intestine in fed and fasted rats. Gastroenterology 1975; 68: 1500-8.

10 Bueno L, Ruckerbusch Y. Migrating myoelectric complexes: disruption enhancement and disorganisation. In: Duthie HL, ed. Gastrointestinal motility in health and disease. Lancaster: MTP Press, 1978: 83-90.

11 Thompson DG. University of London: MD thesis, 1980.

12 Manning AP, Thompson WG, Heaton KW, Morris AF. Towards positive diagnosis of the irritable bowel.
Br Med J 1978; 2: 653-4.

13 Swarbrick ET, Hegarty JE, Bat L, Williams CB, Dawson AM. Site of pain from the irritable bowel. Lancet 1980; 2: 443-6.

14 Moriarty KJM, Dawson AM. Functional abdominal pain: further evidence that whole gut is affected. $\mathrm{Br}$ Med J 1982; 284: 1670-2.

15 Horowitz L, Farrar JT. Intraluminal small intestinal pressures in normal patients and in patients with functional gastrointestinal disorders. Gastroenterology 1962; 42: 455-64.

16 Holdstock DJ, Misiewicz JJ, Waller SL. Observations on the mechanism of abdominal pain. Gut 1969; 10: 19-31. 